\title{
Consumo de frutas, verduras e legumes associado à Síndrome Metabólica e seus componentes em amostra populacional adulta
}

\author{
Consumption of fruit and vegetables associated with the Metabolic \\ Syndrome and its components in an adult population sample
}

Gabriela Kaiser Fullin Castanho ${ }^{1}$

Fabiana Castilho Marsola

Kátia Cristina Portero Mclellan ${ }^{2}$

Marina Nicola ${ }^{2}$

Fernando Moreto ${ }^{1}$

Roberto Carlos Burini

${ }^{1}$ Centro de Metabolismo em Exercício e Nutricão (CeMENutri), Faculdade de Medicina de Botucatu, Universidade Estadual Paulista Júlio de Mesquita Filho (UNESP). Distrito de Rubião Júnior s/n. 18618-970 Botucatu SP gabikaiser@hotmail.com ${ }^{2}$ Departamento de Saúde Pública, Faculdade de Medicina de Botucatu, Universidade Estadual Paulista Júlio de Mesquita Filho (UNESP).

\begin{abstract}
Metabolic Syndrome (MS) increases the risk of cardiovascular events and the recommended intake of fruits and vegetables $(F V)$ is related to its prevention. The scope of this study is to associate the intake of FV and its relation to MS and its components in an adult population sample. A prospective cross-sectional study was conducted with 636 adults in the period between 2004 and 2008. Anthropometric, clinical, dietary and biochemical profiles were recorded for all participants. MS was classified by the NCEP ATP-III, modified for glucose $>100 \mathrm{mg} / \mathrm{dl}$. Logistic regression was used to determine the odds ratio (OR) between the consumption of FV with MS and its components, and the level of significance adopted was 5\%. The intake of fruit had protective effects against obesity (OR: 0.77, CI: 0.38-0.93), hypertriglyceridemia (OR: 0.76, CI: 0.35-0.96) and presence of MS (OR: 0.78, CI: 0.39-0.96), and proper intake of FV showed a protective effect for the presence of MS (OR: 0.79, CI: 0.41-0.95). Vegetable intake did not show protective effects / risk for the presence of MS and its components. The conclusion drawn is that the recommended intake of $F V$ revealed a protective effect against $M S$ and recommended intake of fruit had a protective effect not only for MS but also for its components.
\end{abstract}

Key words Fruit, Vegetables, Metabolic syndrome
Resumo A sindrome metabólica (SM) aumenta o risco de eventos cardiovasculares e o consumo adequado de frutas, verduras e legumes (FVL) está relacionado a sua prevenção. Objetivo: Identificar o consumo de FVL e sua relação com a SM e seus componentes em amostra populacional adulta. Estudo prospectivo de corte transversal com 636 indivíduos adultos, no período de 2004 a 2008. Foram realizadas avaliações antropométrica, clínica, dietética e bioquímica com todos participantes. A SM foi classificada pelo NCEP ATP-III, modificada para a glicose $>100 \mathrm{mg} / \mathrm{dl}$. A regressão logística foi utilizada para verificar a razão de chance entre o consumo de FVL com a SM e seus componentes, e o nivel de significância adotado foi de $5 \%$. O consumo de frutas adequado se mostrou protetor para obesidade abdominal (OR: 0,77; IC: 0,38-0,93), hipertrigliceridemia (OR: 0,76; IC: 0,35-0,96) e presença de SM (OR: 0,78; IC: 0,390,96), e o consumo adequado de FVL apresentou efeito protetor para a presença de SM (OR: 0,79; IC: 0,41-0,95). A análise isolada do consumo adequado de verduras e legumes não mostrou efeitos de proteção/risco para a presença de SM e de seus componentes. O consumo adequado de FVL apresentou efeito protetor para a presença de SM e o consumo adequado de frutas exerceu efeito protetor tanto para a presença de SM e como de seus componentes.

Palavras-chave Frutas, Vegetais, Sindrome metabólica 


\section{Introdução}

A Síndrome Metabólica (SM) é caracterizada por um conjunto de doenças como diabetes mellitus, hipertensão arterial, dislipidemia e obesidade abdominal, aumentando o risco de mortalidade por doenças cardiovasculares. Atualmente, uma das recomendações para prevenção e tratamento da SM é a dieta rica em frutas, vegetais e grãos integrais ${ }^{1}$.

As frutas e os vegetais fazem parte do grupo de alimentos mais importantes no controle do peso e prevenção de doenças ${ }^{2,3}$ e o consumo adequado tem sido associado com risco diminuído de doenças crônicas ${ }^{4-7}$. Porém, o padrão dietético associado a doenças crônicas é caracterizado pelo consumo insuficiente de frutas, verduras e legumes $(\mathrm{FVL})^{8}$, a Organização Mundial de Saúde (OMS) estima que aproximadamente 2,7 milhões de mortes por ano em todo mundo podem ser atribuídas a esse consumo inadequado, estando entre os dez principais fatores de risco para a carga total global de doença em todo o mundo 9 .

A Organização das Nações Unidas sobre Alimentos e Agricultura (Food and Agriculture Organization of the United Nations - FAO) e a OMS recomendam um consumo mínimo de FVL de $400 \mathrm{~g} /$ dia ou o equivalente a cinco porções desses alimentos ${ }^{9}$, já o Ministério da Saúde do Brasil recomenda o consumo diário mínimo de três porções de frutas e quatro de verduras e legumes em seu Guia Alimentar ${ }^{10}$.

Porém, quando são levantados dados sobre o consumo de FVL no mundo os resultados são preocupantes, como nos Estados Unidos que Blanck et al. ${ }^{11}$ avaliaram 1.227.969 adultos nos anos de 1994 e 2005 e verificaram que apenas uma pequena parcela consumiu 5 ou mais porções por dia de FVL, não havendo alteração desse consumo nos dois períodos (homens: 20,6\% vs. $20,3 \%$; mulheres: $28,4 \%$ vs. $29,6 \%$, respectivamente). No Brasil, Jaime e Monteiro ${ }^{12}$ constataram que menos da metade dos indivíduos consumiam verduras e legumes diariamente e, menos de um terço (30\%) reportou o consumo ideal de frutas. No mesmo estudo, apenas $1 / 5$ dos indivíduos relataram consumir FVL todos os dias e apenas $1 / 8$ conhecia a recomendação do consumo de 5 ou mais porções por dia desse grupo de alimentos.

Em relação à associação entre alimentação com a presença de SM, Baxter et al. ${ }^{13}$ notaram que alguns padrões alimentares, como dietas ricas em carnes e grãos refinados, estavam associados com alta incidência de SM, enquanto dietas ricas em frutas, vegetais e grãos integrais foram inversamente associadas com SM. Williams et al. ${ }^{14}$ também verificaram que dieta rica em FVL se correlacionou negativamente com a presença de $S M$, mostrando ser um fator protetor para a mesma. Outros três estudos verificaram que o consumo excessivo de alimentos ricos em gorduras saturadas, gorduras trans, colesterol, açúcares e sal poderia aumentar a prevalência de $\mathrm{SM}^{15-17}$.

Tendo em vista as atuais recomendações nutricionais referentes ao consumo de FVL, o objetivo do presente estudo foi identificar o consumo destes alimentos e sua relação com a SM e seus componentes em amostra populacional adulta.

\section{Casuística e Métodos}

\section{Indivíduos}

Estudo prospectivo de corte transversal com uma amostra populacional composta por 636 (159 homens) indivíduos, de ambos os sexos, acima de 35 anos, triados clinicamente para participar do programa de Mudança do Estilo de Vida (Mexa Pró-saúde) no período de 2004 a 2008. Todos os indivíduos assinaram o Termo de Consentimento Livre e Esclarecido e o trabalho foi aprovado pelo Comitê de Ética em Pesquisa da Faculdade de Medicina da Universidade Estadual Paulista (UNESP).

\section{Avaliação do consumo alimentar}

Em entrevista inicial, os indivíduos foram submetidos à anamnese nutricional e o consumo alimentar foi avaliado por meio da análise do recordatório de 24 horas. Para precisão do consumo dietético foi questionado o horário de cada refeição, tipo de alimento consumido, modo de preparo, quantidade em porções e marca dos produtos. Os dados dietéticos foram convertidos para grama e mililitro a fim de possibilitar a análise química do consumo alimentar, posteriormente foram incluídos no programa de análise nutricional NutWin (2002), versão 1.5.

As preparações culinárias elaboradas com mais de um grupo alimentar tiveram seus ingredientes distinguidos e classificados nos respectivos grupos, procedimento este que segue as recomendações da Pirâmide Alimentar Brasileira Adaptada $^{18}$

No programa foram utilizadas tabelas de referências ${ }^{19-22}$ e rótulos dos alimentos para o cál- 
culo de valor calórico e macronutrientes. Depois de obtido o dado de valor energético total (VET) da alimentação diária referente aos grupos alimentares, calculou-se as porções de frutas, verduras e legumes (FVL) com base na Pirâmide Alimentar Brasileira Adaptada.

A porção de fruta é considerada igual a 70 calorias e o consumo considerado adequado quando $\geq 3$ porções e inadequado $<3$, para a porção de verduras e legumes são 15 calorias e consumo adequado quando $\geq 4$ e inadequado $<$ 4 porções $^{18}$. Para o consumo total de FVL, foi considerado adequado $\geq 5$ porções e inadequado $<5^{9}$.

\section{Circunferência abdominal (CA)}

A circunferência abdominal foi obtida com auxílio de uma fita métrica de metal inelástica de 2,0 m de extensão e com precisão de $0,1 \mathrm{~cm}$, utilizando como referência o ponto médio entre a crista ilíaca e a última costela ${ }^{23}$. Como valores de referências foram utilizados os propostos pela Convenção Latino-americana para Consenso em Obesidade $(1998)^{24}$, considerada aumentada acima de $88,0 \mathrm{~cm}$ para mulheres e $102,0 \mathrm{~cm}$ para homens.

\section{Exames bioquímicos}

Os indivíduos foram submetidas à punção venosa à vácuo padrão para obtenção de amostra sanguínea após jejum noturno de 12 horas. Para obtenção do soro, as amostras foram centrifugadas (3000 rpm - 5 minutos) e em até 4 horas após a coleta sanguínea, as concentrações de glicose, triglicerídios (TG) e HDL-c foram quantificadas no soro pelo método de química seca. A classificação dos valores de normalidade obedeceu a NCEP-ATPIII ${ }^{25,26}$.

\section{Pressão arterial (PA)}

O método utilizado para medida da PA foi o indireto, com técnica auscultatória e esfigmomanômetro de coluna de mercúrio.

Foi avaliada a PA com o indivíduo na posição sentada, seguindo as recomendações da VI Diretriz Brasileira de Hipertensão Arterial (2010) ${ }^{27}$. Foram realizadas três medidas com intervalo de um minuto entre elas, sendo adotada como pressão arterial, a média das duas últimas medidas.

\section{Classificação da Síndrome Metabólica (SM)}

Para a classificação dos indivíduos como portadores ou não de SM e seus componentes, foi utilizada a referência do NCEP ATP- III $(2001)^{25}$ que preconiza o diagnóstico de síndrome metabólica na presença de pelo menos três dos seguintes critérios: circunferência abdominal > $88 \mathrm{~cm}$ para mulheres ou $>102 \mathrm{~cm}$ para homens, concentrações plasmáticas de HDL-c $<50 \mathrm{mg} / \mathrm{dL}$ para mulheres ou $<40 \mathrm{mg} / \mathrm{dL}$ para homens, concentrações plasmáticas TG $>150 \mathrm{mg} / \mathrm{dL}$, pressão arterial (PA) com valores de corte considerando 130/85mmHg e a glicemia de jejum que após sofrer revisão $0^{28}$, diminuiu o valor de glicemia jejum de $>110 \mathrm{mg} / \mathrm{dL}$ para $>100 \mathrm{mg} / \mathrm{dL}$.

\section{Análise estatística}

Para caracterização da amostra foi feita mediana, após ser aplicado o teste Shapiro Wilk para verificação da normalidade (resultados não apresentados), seguido do teste não-paramétrico independente de Mann-Whitney para a comparação entre os gêneros das variáveis quantitativas. Essas análises foram realizadas com o auxílio do programa STATISTICA versão 8.0.

Para verificar a relação entre o consumo de FVL com a SM e seus componentes, foi realizado o Modelo de Regressão Logística (odds ratio) e utilizou-se software SAS for Windows 9.1.3. Foram utilizados dois modelos, sendo o primeiro sem ajuste e o segundo ajustado para gênero e VET. O nível de significância adotado foi de $5 \%$.

\section{Resultados}

A Tabela 1 apresenta as características dos indivíduos estudados. Observou-se que tanto os homens como as mulheres apresentaram consumo inadequado de FVL, porém o coeficiente de variação (CV) mostrou-se extremamente alto. As demais variáveis apresentaram CV dentro da normalidade de $30 \%$, com exceção do triglicerídios. Houve diferença significativa entre os gêneros para CA, PAS, PAD, Glicemia, HDL-c e triglicerídeos.

O consumo de frutas adequado se mostrou protetor para a obesidade abdominal, perdendo efeito após ajuste para sexo e VET, para a hipertrigliceridemia e presença de SM (Tabela 2).

Quando se avaliou isoladamente a associação entre o consumo adequado de verduras e legumes, 
os componentes da SM e a presença de SM, não se observou associação entre as variáveis (Tabela 3).

O estudo de associação entre a presença de $\mathrm{SM}$ e seus componentes alterados e a adequada ingestão de frutas, verduras e legumes mostrou efeito protetor do consumo adequado de FVL para a presença de SM (OR: 0,79; IC: 0,41-0,95), perdendo o efeito de proteção após o ajuste para sexo e VET (OR: 0,82; IC:0,42-1,04) (Tabela 4).

\section{Discussão}

Os resultados do presente estudo mostraram que principalmente as frutas apresentaram fator de proteção contra alguns componentes da SM, corroborando com estudo recente realizado com pacientes brasileiros com Diabetes Mellitos (DM) tipo 2 que demonstrou que o consumo de alimentos ricos em fibras solúveis, representados pelos grãos integrais e frutas, foi um fator de proteção para a presença da $\mathrm{SM}^{29}$. Outro estudo que avaliou o consumo de frutas e doença cardiovascular (DCV) observou uma proteção quando esse consumo era $\geq 3$ porções $/ \mathrm{dia}^{30}$. Perozzo et al. ${ }^{31}$ observaram que o baixo consumo de frutas representou risco tanto para obesidade geral como abdominal.

Esmaillzadeh et al. ${ }^{32}$ também encontraram que o maior consumo de FVL estava associado a menor risco de SM, além de mostrar que os indivíduos que consumiam mais FVL também apresentavam maior quantidade de fibra alimentar, vitamina B6 e magnésio na alimentação.

Tabela 1. Características dos indivíduos e consumo de frutas, verduras e legumes expressos em mediana e coeficiente de variação.

\begin{tabular}{lrrr}
\hline \multicolumn{1}{c}{ Variáveis } & \multicolumn{1}{c}{ Homens } & \multicolumn{1}{c}{ Mulheres } & \multicolumn{1}{c}{ P } \\
\hline Idade (anos) & $50,50(15,0-88,0)$ & $51,5(26,0-85,0)$ & 0,739 \\
Consumo de frutas (porções) & $1,00(0,0-17,00)$ & $1,48(0,0-20,0)$ & 0,825 \\
Consumo de verduras e legumes (porções) & $1,26(0,0-16,50)$ & $1,50(0,0-13,0)$ & 0,485 \\
Consumo de frutas, verduras e legumes (porções) & $3,00(0,0-20,00)$ & $3,00(0,0-24,70)$ & 0,870 \\
Circunferência abdominal (cm) & $99,0(63,0-142,0)$ & $95,0(67,0-138,0)$ & $<0,01$ \\
Pressão Arterial Sistólica (mmHg) & $130,0(96,0-190,0)$ & $121,5(90,0-210,0)$ & $<0,01$ \\
Pressão Arterial Diastólica (mmHg) & $80,0(60,0-120,0)$ & $80,0(60,0-110,0)$ & $<0,01$ \\
Glicemia (mg/dL) & $94,0(50,0-219,0)$ & $90,0(50,0-307,0)$ & $<0,01$ \\
HDL-colesterol (mg/dL) & $43,0(23,0-77,0)$ & $50,0(19,0-90,0)$ & $<0,01$ \\
Triglicerídeos (mg/dL) & $149,0(39,0-521,0)$ & $128,5(36,0-636,0)$ & 0,024 \\
\hline
\end{tabular}

HDL-c $=$ High Density Lipoprotein cholesterol

Tabela 2. Razão de chance entre a adequada ingestão de frutas e a presença de Síndrome Metabólica e seus componentes alterados.

\begin{tabular}{lccccc}
\hline & $\beta$ estimado & SE $(\beta)$ estimado & IC & OR & p valor \\
\hline Circunferência abdominal $(\mathrm{cm})$ & $-0,259$ & 0,115 & $0,38-0,93$ & 0,77 & $0,003^{*}$ \\
& $-0,250^{\text {a }}$ & 0,130 & $0,36-1,00$ & 0,78 & 0,054 \\
Pressão Arterial (mmHg) & 0,027 & 0,113 & $0,68-1,64$ & 1,03 & 0,808 \\
& $0,031^{\text {a }}$ & 0,122 & $0,66-1,72$ & 1,03 & 0,802 \\
Glicemia (mg/dL) & 0,0002 & 0,127 & $0,61-1,64$ & 1,00 & 0,998 \\
& $0,085^{\text {a }}$ & 0,136 & $0,70-2,02$ & 1,09 & 0,532 \\
HDL-colesterol (mg/dL) & $-0,056$ & 0,112 & $0,58-1,39$ & 0,94 & 0,619 \\
& $-0,025^{\text {a }}$ & 0,123 & $0,59-1,54$ & 0,98 & 0,842 \\
Triglicerídeos (mg/dL) & $-0,205$ & 0,117 & $0,42-1,05$ & 0,81 & 0,079 \\
& $-0,274^{\text {a }}$ & 0,130 & $0,35-0,96$ & 0,76 & $0,035^{*}$ \\
Síndrome metabólica & $-0,258$ & 0,108 & $0,39-0,91$ & 0,77 & $0,017^{*}$ \\
& $-0,246^{\text {a }}$ & 0,116 & $0,39-0,96$ & 0,78 & $0,034^{*}$
\end{tabular}

SE: Standard error; OR: Odds Ratio; IC: intervalo de confiança. ${ }^{a}$ : ajustes por valor energético total (VET) e sexo. ${ }^{*} \mathrm{p}<0,05$ 
Tabela 3. Razão de chance entre a adequada ingestão de verduras e legumes e a presença de Síndrome Metabólica e seus componentes alterados.

\begin{tabular}{lccccc}
\hline & $\beta$ estimado & SE $(\beta)$ estimado & IC & OR & p valor \\
\hline Circunferência abdominal $(\mathrm{cm})$ & 0,376 & 0,410 & $0,42-1,58$ & 1,46 & 0,260 \\
& $0,197^{\mathrm{a}}$ & 0,419 & $0,29-7,67$ & 1,22 & 0,637 \\
Pressão Arterial (mmHg) & 0,089 & 0,341 & $0,31-4,55$ & 1,09 & 0,794 \\
& $0,094^{\mathrm{a}}$ & 0,347 & $0,31-4,71$ & 1,10 & 0,786 \\
Glicemia (mg/dL) & 0,492 & 0,327 & $0,74-9,65$ & 1,63 & 0,133 \\
& $0,557^{\mathrm{a}}$ & 0,335 & $0,82-11,32$ & 1,75 & 0,096 \\
HDL-colesterol (mg/dL) & 0,028 & 0,334 & $0,29-3,91$ & 1,03 & 0,934 \\
& $-0,035^{\mathrm{a}}$ & 0,339 & $0,25-3,52$ & 0,97 & 0,918 \\
Triglicerídeos (mg/dL) & 0,049 & 0,344 & $0,24-3,49$ & 0,95 & 0,887 \\
& $0,044^{\mathrm{a}}$ & 0,353 & $0,27-4,35$ & 1,04 & 0,902 \\
Síndrome metabólica & 0,174 & 0,307 & $0,42-4,72$ & 1,19 & 0,571 \\
& $0,158^{\mathrm{a}}$ & 0,310 & $0,41-4,63$ & 1,17 & 0,611 \\
\hline
\end{tabular}

SE: Standard error; OR: Odds Ratio; IC: intervalo de confiança. ${ }^{a}$ : ajustes por valor energético total (VET) e sexo. ${ }^{*} \mathrm{p}<0,05$.

Tabela 4. Razão de chance entre a adequada ingestão de frutas, verduras e legumes e a presença de Síndrome Metabólica e seus componentes alterados.

\begin{tabular}{lccccc}
\hline & $\beta$ estimado & SE $(\beta)$ estimado & IC & OR & p valor \\
\hline Circunferência abdominal $(\mathrm{cm})$ & $-0,163$ & 0,114 & $0,46-1,13$ & 0,85 & 0,153 \\
& $-0,137^{\text {a }}$ & 0,129 & $0,46-1,26$ & 0,87 & 0,290 \\
Pressão Arterial (mmHg) & $-0,122$ & 0,111 & $0,51-1,21$ & 0,88 & 0,273 \\
& $-0,109^{\text {a }}$ & 0,121 & $0,50-1,29$ & 0,90 & 0,368 \\
Glicemia (mg/dL) & 0,067 & 0,125 & $0,70-1,87$ & 1,07 & 0,589 \\
& $0,184^{\text {a }}$ & 0,134 & $0,85-2,45$ & 1,20 & 0,170 \\
HDL-colesterol (mg/dL) & $-0,193$ & 0,112 & $0,44-1,05$ & 0,82 & 0,084 \\
& $-0,175^{\text {a }}$ & 0,122 & $0,44-1,14$ & 0,84 & 0,151 \\
Triglicerídeos (mg/dL) & $-0,055$ & 0,116 & $0,57-1,41$ & 0,95 & 0,634 \\
& $-0,094^{\text {a }}$ & 0,127 & $0,50-1,36$ & 0,91 & 0,458 \\
Síndrome metabólica & $-0,237$ & 0,107 & $0,41-0,95$ & 0,79 & $0,027^{*}$ \\
& $-0,203^{\text {a }}$ & 0,115 & $0,42-1,04$ & 0,82 & 0,076 \\
\hline
\end{tabular}

SE: Standard error; OR: Odds Ratio; IC: intervalo de confiança. ${ }^{a}$ : ajustes por valor energético total (VET) e sexo. ${ }^{*}$ p $<0,05$

Diversos estudos epidemiológicos demonstraram que o consumo de FVL pode ser associado com menor incidência e mortalidade relacionadas a problemas de saúde como obesidade, hipertensão, DM, SM e DCV ${ }^{33-36}$. Esse estudo falhou em demonstrar associações entre o consumo de verduras e legumes e a presença de SM e seus componentes. Este fato pode ser explicado pelo inadequado consumo destes alimentos pela população estudada, podendo representar um viés importante na análise dos dados.

Quando somados o consumo de frutas, verduras e legumes e associados à presença de SM, os resultados mostraram que existe um efeito protetor, evidenciando que a ingestão recomendada de 5 porções de FVL pode ser um fator de proteção para a presença de SM. Este efeito se perdeu quando a associação foi ajustada para sexo e VET. Essa perda da significância estatística pode estar relacionada ao baixo consumo destes alimentos (FVL) pela população estudada.

O presente estudo mostrou resultados positivos do consumo de frutas e a presença da SM, apesar das verduras e legumes não mostrarem efeitos sobre a doença, muitos estudos comprovaram sua eficácia tanto em componentes da SM 
como em outras doenças relacionadas, como DM e DCV. Quando se associou a ingestão de FVL e respeitou-se a recomendação de $400 \mathrm{~g}$ ou 5 porções/dia, também houve diminuição do risco para SM ou outras comorbidades, como visto em algumas populações de diversos estudos.

Uma pesquisa que avaliou a ingestão de 5 porções por dia de FVL observou proteção dessa para as $\mathrm{DCV}^{30}$. Nothlings et al. ${ }^{37}$ relataram que apenas incrementando a alimentação com $80 \mathrm{~g} /$ dia de FVL pode-se diminuir em $6 \%$ o risco de todas as causas de mortalidade. Analisadas separadamente, a ingestão de verduras e legumes foi também associada inversamente com todas as causas de morte e com mortes para DCV numa população européia diabética.

Em estudo com 77.891 japoneses, aqueles que apresentaram maior consumo de frutas, porém não vegetais, tiveram associação com menor risco de DCV, resultados parecidos com os mostrados nesse estudo $(40)^{38}$. Esmaillzadeh et al. ${ }^{32}$ avaliaram 486 mulheres iranianas entre 40-60 anos e verificaram uma tendência inversa entre ingestão de frutas e a probabilidade de ter SM. Entretanto, nenhuma associação significante foi observada entre frutas e menor HDL-c. O maior consumo de vegetais foi também associado com menor risco de SM.

Assim como visto no presente estudo, os resultados de McNaughton et al. ${ }^{39}$ com 1.265 pessoas, de 36 a 53 anos, também sugeriram uma associação inversa do consumo de FVL com CA em mulheres. Em Chennai (Índia), numa amostra de 983 indivíduos com média de 20 anos, o maior consumo de FVL também mostrou uma associação inversa significativa com PA, CA, colesterol total e LDL-c quando comparado com menor consumo ${ }^{40}$.

Portanto, como visto neste estudo e em outros citados, o consumo de FVL, isolados ou associados, é um fator de proteção para o desenvolvimento de SM, DM, DCV, dislipidemias e outras causas de mortes, porém a maioria das populações, inclusive a deste estudo, não tem alcançado a ingestão recomendada desses alimentos, sendo o incentivo a melhor forma de estimular esse consumo, devendo ser feito para todos os indivíduos.

\section{Conclusão}

Com os resultados obtidos foi observado que o consumo de frutas teve efeito protetor para a circunferência abdominal alterada, hipertrigliceridemia e presença de Síndrome Metabólica. Porém, assim como no presente estudo e em outros citados, o consumo de frutas, verduras e legumes é abaixo do recomendado, sendo necessário incentivo à população para a melhora da ingestão desse grupo de alimentos.

\section{Colaboradores}

GKF Castanho, FC Marsola e M Nicola contribuíram na concepção, delineamento, análise e interpretação dos dados, redação do artigo e aprovação da versão a ser publicada. KCP Mclellan contribuiu na redação do artigo e aprovação da versão a ser publicada. F Moreto contribuiu na concepção, delineamento dos dados. RC Burini contribuiu no delineamento, análise e interpretação dos dados e aprovação da versão a ser publicada. 


\section{Referências}

1. Lichtenstein. Diet and lifestyle recommendations revision 2006: a scientific statement from the american heart association nutrition committee. Circulation 2006; 114(1):82-96.

2. Knai C, Pomerleau J, Lock K, McKee M. Getting children to eat more fruit and vegetables: A systemic review. Prev Med 2006; 42(2):85-95.

3. Singh M. Role of micronutrients for physical growth and mental development. Indian J Pediatr 2004; 71(1):59-62.

4. Dauchet L, Amouyel P, Hereberg S, Dallongeville J. Fruit and vegetable consumption and risk of coronary heart disease: A meta-analysis of cohort studies. J Nutr 2006; 136(10):2588.

5. Bertsias G, Linardakis M, Mammas I, Kafatos A. Fruit and vegetable consumption in relation to health and diet of medical students in Crete, Greece. Int J Vitam Nutr Res 2005; 75(2):107-117.

6. Pavia M, Pileggi C, Nobile CG, Angelillo IF. Association between fruit and vegetable consumption and oral cancer: A meta-analysis of observational studies. Am J Clin Nutr 2006; 83(5):1126-1134.

7. Prynne CJ, Mishra GD, O’Connell MA, Muniz G, Laskey MA, Yan L, Prentice A, Ginty F. Fruit and vegetable intakes and bone mineral status: A cross sectional study in 5 age and sex cohorts. Am J Clin Nutr 2006; 83(6):1420-1428.

8. World Health Organization (WHO). The world health report 2002: reducing risks, promoting healthy life. WHO: Geneva; 2002.

9. World Health Organization (WHO). Diet, nutrition and the prevention of chronic diseases. report of a joint WHO/FAO expert consultation. Geneva: WHO; 2003. (Technical Report Series, n. 916)

10. Brasil. Ministério da Saúde (MS). Secretaria de Atenção à Saúde. Coordenação-Geral da Política de Alimentação e Nutrição. Guia Alimentar para a População Brasileira: promovendo a alimentação saudável. Brasília: MS; 2005. (Série A. Normas e Manuais Técnicos)

11. Blanck HM, Gillespie C, Kimmons JE, Seymour, JD, Serdula, MK. Trends in fruit and vegetable consumption among U.S. men and women, 1994-2005. Prev Chronic Dis 2008; 5(2):A35.

12. Jaime PC, Monteiro CA. Fruit and vegetable intake by Brazilian adults, 2003. Cad Saude Publica 2005; 21(Supl.):S19-S24.

13. Baxter AJ, Coyne T, McClintock C. Dietary patterns and metabolic syndrome - a review of epidemiologic evidence. Asia Pac J Clin Nutr 2006; 15(2):134142.

14. Williams DE, Prevost At, Whichelow MJ, Cox, BD, Day, NE, Wareham, NJ. A cross-sectional study of dietary patterns with glucose intolerance and others features of the metabolic syndrome. Br J Nutr 2000; 83(3):257-266

15. American Diabetes Association(ADA). Clinical practice recommendations. Diabetes Care 2004; 27(1): S141-S143

16. Mozaffarian D, Pischon T, Hankinson S, Rifai N, Joshipura K, Willett W, Rimm, EB. Dietary intake of trans fatty acids and systemic inflammation in women. Am J Clin Nutr 2004; 79(4):606-612.
17. Rique ABR, Soares EA, Meirelles CM. Nutrição e exercício na prevenção e controle das doenças cardiovasculares. Rev Bras Med Esp. 2002; 8(6):1-11.

18. Philippi ST, Latterza AR, Cruz ATR, Ribeiro LC. Adapted food pyramid: a guide for a right food choice. Rev Nutr. 1999; 12(1):65-80.

19. Universidade Estadual de Campinas (Unicamp). Tabela de Composição de Alimentos - Versão 2, Campinas: Unicamp; 2006. [acessado 2011 jun 11]. Disponível em: http://www.unicamp.br/nepa/taco/.

20. Phillippi ST. Tabela de Composição de Alimentos: suporte para Decisão Nutricional. 2a Edição. São Paulo: Coronário; 2002.

21. Pinheiro ABV, Lacerda EMA, Benzecry EH, Gomes MCS, Costa VM. Tabela para avaliação de consumo alimentar em medidas caseiras. 5a Edição. São Paulo: Atheneu; 2004

22. Instituto de Brasileiro de Geografia e Estatística (IGBE). Tabela de Composição de Alimentos, 5ª Edição. Rio de Janeiro: IBGE; 1999. [acessado 2011 jun 26]. Disponível em: http://biblioteca.ibge.gov.br/.

23. Heyward VH, Stolarczyk LM. Avaliação da composição corporal aplicada. 1 ${ }^{\text {a }}$ Edição. Barueri: São Paulo; 2000.

24. Convenção Latino-Americana para Consenso em Obesidade (CLACO). Consenso Latino-Americano em Obesidade, Rio de Janeiro, Brasília: MS; 1998.

25. Executive Summary of The Third Report of The National Cholesterol Education Program (NCEP) Expert Panel on Detection. Evaluation, And Treatment of High Blood Cholesterol In Adults (Adult Treatment Panel III). Jama 2001; 285(19):2486-2497.

26. National Cholesterol Education Program (NCEP) Expert Panel on Detection, Evaluation, and Treatment of High Blood Cholesterol in Adults (Adult Treatment Panel III). Third Report of the National Cholesterol Education Program (NCEP) Expert Panel on Detection, Evaluation, and Treatment of High Blood Cholesterol in Adults (Adult Treatment Panel III) final report Circulation 2002; 106(25):3143-3421.

27. Sociedade Brasileira de Cardiologia(SBC), Sociedade Brasileira de Hipertensão(SBH), Sociedade Brasileira de Nefrologia(SBN).VI Diretrizes Brasileiras de Hipertensão. Arq Bras Cardiol 2010; 95(Supl. 1):1-51.

28. Grundy SM, Brewer Júnior HB, Cleeman JI, Smith Júnior SC, Lenfant C; National Heart, Lung, Blood Institute, American Heart Association. Definition of metabolic syndrome: report of the National Heart, Lung, and Blood Institute/American Heart Association conference on scientific issues related to definition. Arterioscler Thromb Vasc Biol 2004; 24(2):e13-18.

29. Steemburgo T, Dall'Alba V, Almeida JC, Silva FM, Zelmanovitz T, Gross JL. Intake of fibers from fruits and whole grains has a protective role for the presence of metabolic syndrome in patients with type 2 diabetes. The Endocrine Society's 88th Annual Meeting (ENDO); 2006 Jun 24-27, Boston, Massachusetts, USA; 2006. 
30. Hung H, Joshipura KJ, Jiang R, Hu FB, Hunter D, Smith-Warner SA, Colditz GA, Rosner B, Spiegelman D, Willet WC. Fruit and Vegetable Intake and Risk of Major Chronic Disease. J Natl Cancer Inst 2004; 96(21):1577-1584.

31. Perozzo G, Olinto MTA, Dias-da-Costa, JS, Henn RL, Sarriera J, Patussi MP. Associação dos padrões alimentares com obesidade geral e abdominal em mulheres residentes no Sul do Brasil. Cad Saude Publica 2008; 24(10):2427-2439.

32. Esmaillzadeh A, Kimiagar M, Mehrabi Y, Azadbakht L, Hu FB, Willett WC. Fruit and vegetable intakes, C-reactive protein, and the metabolic syndrome1-3. Am J Clin Nutr 2006; 84(6):1489-1497.

33. He K, Hu FB, Colditz GA, Manson JE, Willett WC, Liu S. Changes in intake of fruits and vegetables in relation to risk of obesity and weight gain among middleaged women. Int J Obes Relat Metab Disord 2004; 28(11):1569-1574.

34. Bazzano LA. The high cost of not consuming fruits and vegetables. J Am Diet Assoc 2006; 106:13641368.

35. Genkinger JM, Platz EA, Hoffman SC, Comstock GW, Helzlsouer KJ. Fruit, vegetable, and antioxidant intake and all-cause, cancer, and cardiovascular disease mortality in a community-dwelling population in Washington County, Maryland. Am J Epidemiol 2004; 160(12):1223-1233.

36. Bazzano LA, He J, Ogden LG, Loria CM, Vupputuri S, Myers L, Whelton PK. Fruit and vegetable intake and risk of cardiovascular disease in US adults: the first National Health and Nutrition Examination Survey Epidemiologic Follow-up Study. Am J Clin Nutr 2002; 76(1):93-99.
37. Nothlings U, Schulze MB, Weikert C, Boeing H, Van der Schouw YT, Bamia C, Benetou V, Lagiou P, Krogh V, Beulens JWJ, Peeters PHM, Halkjaer J, Tjonneland N, Tumino R, Panico S, Malasa G, Clavel-Chapelon F, de Lauzon B, Boutron-Ruault M, Vercambre M, Kaaks R, Linseisen J, Overvad K, Arriola L, Ardanaz A, Gonzalez CA, Tormo MJ, Bingham S, Khaw K, Key TJA, Vineis P, Riboli E, Ferrari P, Boffetta P, Bueno-de-Mesquita HB, Van der A DL, Berglund G, Wirfält E, Hallmans G, Johansson I, Lund E, Trichopoulo A. Intake of Vegetables, Legumes, and Fruit, and Risk for All-Cause, Cardiovascular, and Cancer Mortality in a European Diabetic Population. I Nutr 2008; 138(4):775-781.

38. Takachi R, Inoue $\mathrm{M}$, Ishihara J, Kurahashi N, Iwasaki M, Sasazuki S, Iso H, Tsubono Y, Tsugane S, JPHC Study Group. Fruit and Vegetable Intake and Risk of Total Cancer and Cardiovascular Disease Japan Public Health Center-based Prospective Study. Am J Epidemiol. 2008; 167(1):59-70.

39. McNaughton SA, Mishra GD, Stephen AM, Wadsworth ME. Dietary patterns throughout adult life are associated with body mass index, waist circumference, blood pressure, and red cell folate. J Nutr. 2007; 137(1):99-105

40. Radhika G, Sudha V, Sathya RM, Ganesan A, Mohan V. Association of fruit and vegetable intake with cardiovascular risk factors in urban south Indians. Br J Nutr. 2008; 99(2):398-405.

Artigo apresentado em 14/04/2011

Aprovado em 29/08/2011

Versão final aprovada em 31/01/2012 